\title{
Short-term Effects of Transcranial Near-Infrared Photobiomodulation on Motor Performance in Healthy Human Subjects: An Experimental Single- Blind Randomized Clinical Trial
}

\author{
Atefeh Fekri', Ali Jahan ${ }^{2}$, Maryam Moghadam Salimi ${ }^{1}$, Ali E. Oskouei ${ }^{{ }^{*}}$ \\ ${ }^{1}$ Department of Physiotherapy, Faculty of Rehabilitation, Tabriz University of Medical Sciences, Tabriz, Iran \\ ${ }^{2}$ Department of Speech Therapy, Faculty of Rehabilitation, Tabriz University of Medical Sciences, Tabriz, Iran \\ ${ }^{3}$ Physical Medicine and Rehabilitation Research Center, Tabriz University of Medical Sciences, Tabriz, Iran
}

\section{*Correspondence to Ali E. Oskouei, \\ $\mathrm{PhD}$, Department of Physiotherapy, Physical Medicine and Rehabilitation Research Center, Tabriz University of Medical Sciences, Tabriz, Iran. Tel: +98(41)33371997; Fax: +98(41) 33375359; Email: eterafoskouei@tbzmed.ac.ir}

Published online October 1, 2019

\begin{abstract}
Introduction: Transcranial near-infrared photobiomodulation (NIR-PBM) is a new noninvasive procedure which transcranially applies a near-infrared wavelength to the scalp with a laser or a light-emitting diode (LED) source. Improvement in the neurological or psychological symptoms has been reported following light irradiation. However, to our knowledge, there is no study to investigate the effects of transcranial NIR-PBM on motor performance directly. Therefore, the objective of this study was to investigate the short-term effects of transcranial NIR-PBM on motor performance in healthy human subjects.

Methods: In this experimental single-blind randomized clinical trial study, 56 right-handed healthy participants, whose ages ranged from 18 to 30, were randomly assigned to (1) Real transcranial NIR-PBMC3 group ( $n=14)$, (2) Sham transcranial NIR-PBMC3 group $(n=14)$, (3) Real transcranial NIR-PBMC4 group $(n=14)$, and (4) Sham transcranial NIR-PBMC4 group $(n=14)$. We applied the $808 \mathrm{~nm}$ laser with irradiation energy density of $60 \mathrm{~J} / \mathrm{cm}^{2}$ and power density of $200 \mathrm{mw} / \mathrm{cm}^{2}$ to the C3 or C4 points of the scalp. The number of finger taps as an indicator of motor performance was assessed by the finger-tapping test (FTT) before and after irradiation of transcranial NIR-PBM on the corresponding points of the scalp for 5 minutes.

Results: The results showed that the number of finger taps in both right and left hands following the use of transcranial NIR-PBM in the real transcranial NIR-PBMC3 group significantly increased $(P<0.05)$.

Conclusion: We concluded that using transcranial NIR-PBM with a laser source on C3 point of the motor cortex in right-handed healthy people can increase the number of finger taps in both hands as an indicator of motor performance improvement.

Keywords: Transcranial; Cytochrome-c oxidase; Laser; Motor cortex.
\end{abstract}

\section{Introduction}

Transcranial near-infrared photobiomodulation (NIRPBM) consists of the wavelength of $600-1100 \mathrm{~nm}$ and is an innovative procedure in which red to near-infrared light irradiation is applied transcranially to the interesting area of the scalp. ${ }^{1}$ Lasers or light-emitting diodes (LEDs) are considered as two sources of light irradiation in PBM. ${ }^{2}$ Light penetration into the brain tissue in this wavelength has been established in previous studies on cadaver as well as computerized models..$^{3-6}$ One mechanism of PBM is associated with the potential for increasing cellular energy production by modulating mitochondrial cytochrome-c oxidase. $^{7,8}$ It has also been suggested that PBM may be associated with an increase in the brain focal cerebral blood flow by releasing nitric oxide and modifying cell gene expression. ${ }^{1,3,9-13}$

Many studies investigated the effectiveness of the transcranial NIR-PBM in clinical and normal conditions. ${ }^{14-18}$ Improvement in the neurological or psychological symptoms has been reported after light irradiation in both animal and human models. ${ }^{3,19-24}$ The promotion of cognitive performance such as the reaction time has been observed in some cognitive studies assessed by the attentional tests or the psychomotor vigilance task (PVT). ${ }^{20,25,26}$ Moreover, some improvements in motor function, balance, coordination and muscle power in traumatic brain injury, stroke, and Parkinson diseases were reported as the accessory findings of the PBM

Please cite this article as follows: Fekri A, Jahan A, Moghadam Salimi M, Oskouei AE. Short-term effects of transcranial near-infrared photobiomodulation on motor performance in healthy human subjects: an experimental single-blind randomized clinical trial. $J$ Lasers Med Sci. 2019;10(4):317-323. doi:10.15171/jlms.2019.51. 
studies on animal models after modulating the frontal or pre-frontal regions. ${ }^{27-32}$ However, to our knowledge, none of these available studies targeted motor performance as the main purpose of the transcranial NIR-PBM. Needless to say, all these few studies with the implication of motor improvement have been performed on animal models. Since the previous studies approved the utility and safety of the transcranial NIR-PBM in humans with normal and clinical conditions, we decided to investigate the effects of this method on motor function by directly irradiating the primary motor cortex in human subjects. We hypothesized that transcranial light irradiation may improve motor performance in healthy subjects.

\section{Methods}

The procedure was performed at the Faculty of Rehabilitation, Tabriz University of Medical Sciences. Fifty-six right-handed healthy young adults, whose ages ranged from 18 to 30 , were recruited. The Persian version of the Chapman questionnaire with the reliability score (0.92) was used in the present study to determine the participants' handedness. ${ }^{33,34}$ The exclusion criteria were the history of orthopedic or neurologic disease of upper extremities, left- handedness, uncorrected visual impairment, sleep disorders, pregnancy, epilepsy, regular manual activity involving high dexterity (such as playing the piano), neurological or psychological disorders, medications that affect the neural system, and a high rate of errors in doing the task..$^{25,35-37}$ The participants were informed of the study purpose and the experimental procedure.

Light Irradiation Procedure

The participants were assigned to 4 groups using a simple random allocation strategy and a random number list: (1)
Real transcranial NIR-PBMC3 group $(\mathrm{n}=14)$ in which the laser light with $60 \mathrm{~J} / \mathrm{cm}^{2}$ energy density was irradiated on C3 point of the skull (located on the left primary motor cortex) according to the 10-20 system of the Electroencephalography (EEG), (2) Sham transcranial NIR-PBMC3 group $(n=14)$ in which the laser light was irradiated on $\mathrm{C} 3$ point of the skull using a filter between the laser applicator and the participants' scalp to ensure that they received no laser energy, (3) Real transcranial NIR-PBMC4 group $(n=14)$ in which the laser light with $60 \mathrm{~J} / \mathrm{cm}^{2}$ energy density was irradiated on $\mathrm{C} 4$ point of the skull (located on the right primary motor cortex), and (4) Sham transcranial NIR-PBMC4 group $(n=14)$ in which the laser light was irradiated on the $\mathrm{C} 4$ point of the skull using a filter between the laser applicator and the participants' scalp to ensure that the participants received no laser dosage (Figure 1). All participants received laser irradiation for 5 minutes, which was calculated by a formula.

The Laser Lumix 3 Plus Ultra model (Italy, Fisioline) with an $808 \mathrm{~nm}$ wavelength, continuous waves, and a circular cross-section $\left(3.14 \mathrm{~cm}^{2}\right)$ was used for light irradiation. The laser machine was calibrated by an internal calibrator before starting the experiment. The amount of the power density in this study was $200 \mathrm{mw} /$ $\mathrm{cm}^{2}\left(628 \mathrm{mw} / 3.14 \mathrm{~cm}^{2}=200 \mathrm{mw} / \mathrm{cm}^{2}\right)$ and the energy density was $60 \mathrm{~J} / \mathrm{cm}^{2}$, which were safe with no physical tissue damage, except allowable heat according to the previous studies. The treatment time was determined as 5 minutes or 300 seconds $\left(0.2 \mathrm{~W} / \mathrm{cm}^{2 *} 300 \mathrm{~s}=60 \mathrm{~J} / \mathrm{cm}^{2}\right) .^{20,38}$

Motor Task

Motor performance was assessed by the finger-tapping test (FTT) immediately before and after receiving the light irradiation. The finger tapping test is a neuropsychological

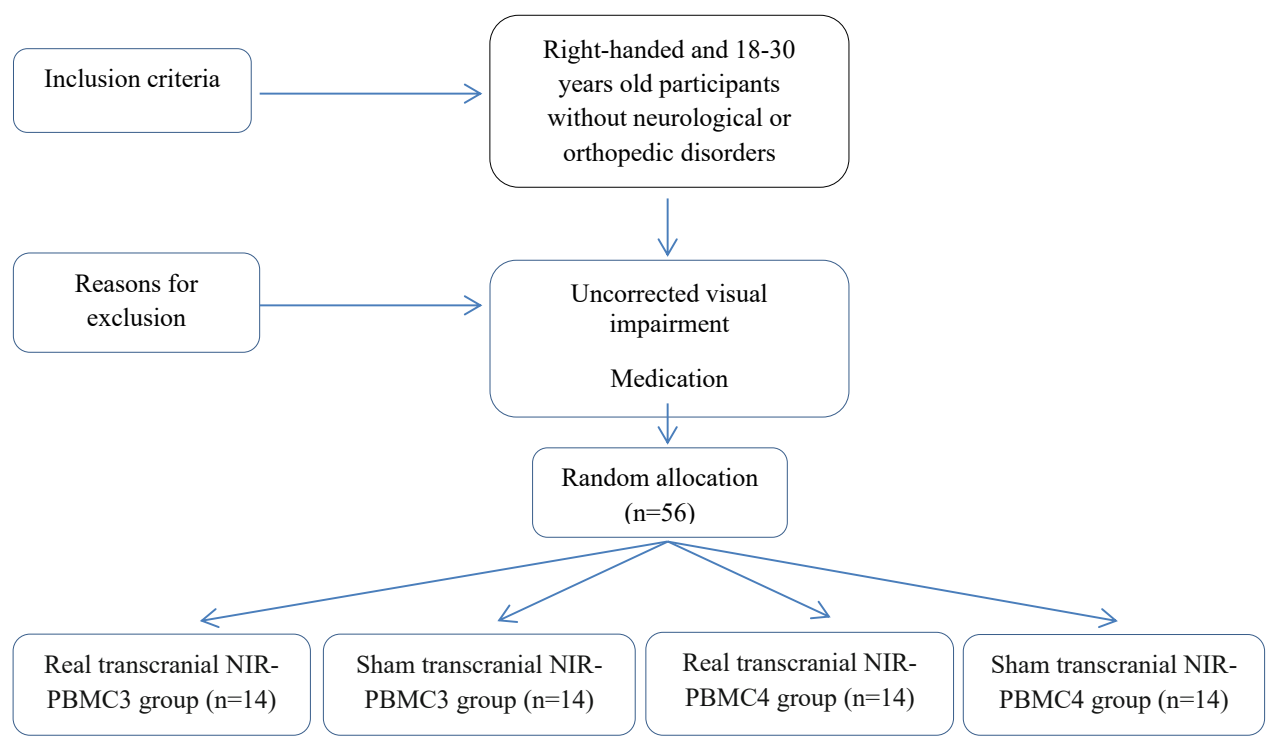

Figure 1. Flowchart of the Study Design. 
test that assesses motor speed, coordination, attention and alertness as motor control parameters and it can assess the integrity of the neuromuscular system too. In this test, the participants seated themselves comfortably on a chair with the forearms rested on a table in front of them. Both elbows were flexed at $90^{\circ}$. The right index finger was then placed on a key that was prepared on the flat board. After initial preparation and familiarization, the participants were asked to tap the key as quickly as possible by flexing and extending the metacarpophalangeal joint of the index finger. The participants performed 5 trials of the tapping task with the dominant hand (right hand). The procedure was then repeated with the non-dominant hand (left hand). Five 10-second trials were given for each hand except when the results were too variable from one trial to another. Specifically, the test procedure required that the five serial trials for each hand be within a 5-point range from the fastest to slowest tapping task. If one or more of the trials exceeded this range, additional trials were given and the scores of the unexpected trials were rejected. A maximum of 10 trials with each hand was allowed in order to prevent any possible fatigue effects with a rest period of 10 seconds between trials. The average number of finger tapping in five 10-second trials was taken as the mean of finger tapping score calculated for each hand and it was used for the statistical analysis. ${ }^{39,40}$

\section{Statistical Analysis}

Statistical analysis was performed using Statistical Package for the Social Sciences version 23.0 (SPSS, Chicago, IL, USA). Two different series of repeated measures ANOVA (RANOVA) were performed for within-group analysis in each group (NIR-PBMC3/NIR-PBMC4 irradiation). Condition (Real/Sham) and sex were considered as between-subject variables and time (pre/post) as a within-subject variable. As the sphericity assumption was violated, Greenhouse-Geisser values were reported. Interaction of time by condition was hypothesized as the effectiveness of the transcranial NIR-PBM. The Kolmogorov-Smirnov test was utilized to study the data distribution, and random allocation of participants was checked by multivariate ANOVA.

The significance level was set at 0.05 . All analyses were based on the per-protocol approach. The participants fully accomplished the study and there were no missing data.

\section{Results}

Based on the pilot study $(n=7)$ and the test of the two independent groups, the sample size was calculated by $\mathrm{G}^{*}$ Power freeware. The effect size was 1.18 (pre/post mean difference for real $=2.4 \pm 1.9$ and sham $=0.11 \pm 1.9$ conditions) in the right hand after C3 irradiation. Given $\alpha=0.05$ and $\beta=0.2$, the total size was determined to be 56 participants (14 participants for each group). Descriptive statistics of the participants' characteristics are presented in Table 1. The K-S test revealed normal distribution of data in 4 groups $(P>0.05)$. The multivariate ANOVA could not show a significant difference between pre-PBM hand performance in all participants, which justifies the random allocation of participants [Pillai's $\mathrm{F}(6,104)=0.45$, $P=0.84]$. The main effect of the hand was significant in favor of the right hand $(P>0.05)$.

There was a significant interaction between condition and time $[F(1,26)=6.09, p=0.02$, eta $=0.19]$ in the real transcranial NIR-PBMC3 group. In other words, the number of finger taps in this group following laser irradiation significantly increased from 57.63 to 60.34 and 52.13 to 53.74 in the right hand and the left hand respectively $(P<0.05)$. However, there was no statistically significant increase in the number of finger taps in both hands in the sham transcranial NIR-PBMC3 group (Figure 2). The interactions of condition $\times$ time $\times$ hand and condition $\times$ time $\times \operatorname{sex} \times$ hand were not significant $(P>0.05)$.

In transcranial NIR-PBMC4 groups, none of the interactions were significant $(P>0.05)$. In other words, the number of finger taps in these groups following laser irradiation was similar in real and sham conditions (Figure 3). Percentage improvement in the number of finger taps of the study participants in all study groups is shown in Table 2.

\section{Discussion}

This study was the first study which investigated the effectiveness of the transcranial NIR-PBM with a laser source $\left(808 \mathrm{~nm}, 60 \mathrm{~J} / \mathrm{cm}^{2}\right.$, and $\left.200 \mathrm{mw} / \mathrm{cm}^{2}\right)$ in motor performance by direct applications to the motor region of

Table 1. Descriptive Statistics (Mean \pm SD) of the Participants' Characteristics in Real Transcranial NIR-PBMC3, Sham Transcranial NIR-PBMC3, Real Transcranial NIR-PBMC4, and Sham Transcranial NIR-PBMC4 Groups

\begin{tabular}{|c|c|c|c|c|c|}
\hline Characteristics & $\begin{array}{c}\text { Real Transcranial } \\
\text { NIR-PBMC3 }\end{array}$ & $\begin{array}{c}\text { Sham Transcranial } \\
\text { NIR-PBMC3 }\end{array}$ & $\begin{array}{c}\text { Real Transcranial } \\
\text { NIR-PBMC4 }\end{array}$ & $\begin{array}{c}\text { Sham Transcranial } \\
\text { NIR-PBMC4 }\end{array}$ & $P$ Value \\
\hline Sex (male, \%) & 22.8 & 22.8 & 28.5 & 25.7 & 0.8 \\
\hline Age $(y)$ & $21.2 \pm 3.1$ & $19.9 \pm 1.7$ & $20.6 \pm 1.3$ & $21.6 \pm 3.3$ & 0.2 \\
\hline Height $(\mathrm{cm})$ & $170.9 \pm 9.2$ & $173.4 \pm 12.0$ & $171.5 \pm 8.7$ & $170.4 \pm 10.7$ & 0.8 \\
\hline Weight (kg) & $66.6 \pm 11.0$ & $64.8 \pm 14.2$ & $64.2 \pm 11.4$ & $68.6 \pm 11.2$ & 0.6 \\
\hline $\operatorname{BMI}\left(\mathrm{kg} / \mathrm{m}^{2}\right)$ & $22.1 \pm 2.7$ & $20.8 \pm 2.4$ & $21.2 \pm 2.8$ & $23.2 \pm 2.5$ & 0.05 \\
\hline
\end{tabular}

Abbreviation: BMI, body mass index 

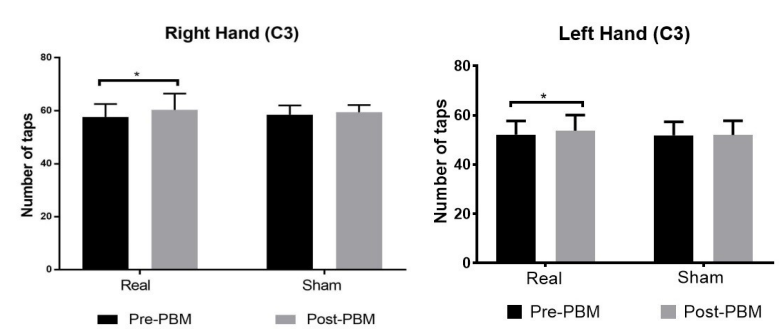

Figure 2. The Mean Number of Finger Taps in Real Transcranial NIR-PBMC3 and Sham Transcranial NIR-PBMC3 Groups for Left and Right Hands. ${ }^{*} P<0.05$.

the human's cortex. The findings showed that increasing the number of finger taps in FTT may serve as an indicator of motor control and motor performance, which could result from improving the neuronal function of the motor region and modulating neural cells after the use of the NIR-PBM. In other words, motor speed is one of the motor control and motor performance parameters which can be assessed via FTT. ${ }^{39,40}$ Therefore, any increase in the number of taps or speed might be associated with motor performance and modulation of neural cells.

Similar to our study, some previous studies reported improvement of motor control and performance after the use of the transcranial NIR-PBM in healthy and neurological conditions. They showed that transcranial light irradiation could improve balance, coordination and motor function in animal studies after using the transcranial NIR-PBM on cognitive regions of the brain. They also reported that light therapy could promote muscle power after its applying to pre-frontal and frontal regions. According to these studies, a decrease in brain lesion size and active clot dose and an increase in neuroprotective factors after light therapy were the main causes of functional improvement as an objective result. On the basis of these studies, the effect of PBM mechanism on brain function seems to be associated with the special biochemical changes in neural cells. ${ }^{27-32}$ The light therapy could increase the adenosine triphosphate (ATP) and nitric oxide by activating the cytochrome-c oxidase. These changes lead to an increase in metabolic capacity, energy production, and cerebral blood flow., ${ }^{1,3}$, $7-12,18,23,24$ Therefore, it may imply that improving motor performance in FTT in our study could be the result of the similar biochemical changes in neural cells of motor cortex after the use of the transcranial NIR-PBM.

Our results showed the improvement of motor
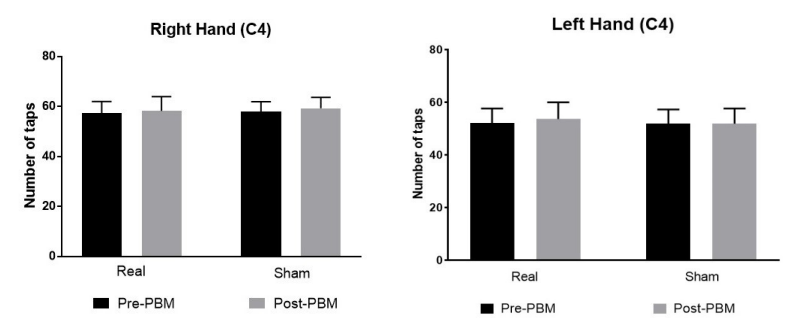

Figure 3. The Mean Number of Finger Taps in Real Transcranial NIR-PBMC4 and Sham Transcranial NIR-PBMC4 Groups for Left and Right Hands.

performance in both right and left hands of the participants after applying the transcranial NIR-PBMC3 to the left primary motor cortex. Also, we expected that the modulation of right motor cortex or $\mathrm{C} 4$ could improve the motor function in the contralateral (left) hand, but the result was not statistically significant. So, it demonstrates that only cellular biochemical changes cannot explain these findings. To complete our discussion, we can refer to the human's cerebral hemispheric lateralization theory. According to this theory, right-handed people have a more excitable corticospinal system in the left hemisphere than in the right side. ${ }^{41-44}$ In addition, the left hemisphere is generally dominant for language and skilled movements. ${ }^{41,42,45,46}$ and has a bilateral effect on motor control of both hands in both left- and righthanded individuals. ${ }^{47}$ In our study on right-handed individuals, accidentally, the excitable hemisphere and the dominance hemisphere for skilled movements were the same. So, the activation of the left hemisphere by using the transcranial NIR-PBM in right-handed people promoted motor function bilaterally through FTT as it was observed in this study.

To investigate that which parameter is more important (activating the excitable hemisphere or just activating the left hemisphere as a dominance hemisphere for skilled movements), we need another research with a similar design in left-handed individuals.

The most important limitation in this field of studies is the choice of effective laser irradiation dosage for penetrating and modulating primary motor cortex. There is not solidarity on specific effective dose in transcranial NIR-PBM studies. We just used the safe and penetrable laser dosage according to the previous studies. Since the FTT is a compound task and it can assess the motor and cognitive (like attention) aspects, ${ }^{39,40}$

Table 2. Percentage Improvement in the Number of Finger Taps of the Study Participants in Real Transcranial NIR-PBMC3, Sham Transcranial NIR-PBMC3, Real Transcranial NIR-PBMC4, and Sham Transcranial NIR-PBMC4 Groups After Irradiation

\begin{tabular}{|c|c|c|c|c|}
\hline Condition & $\begin{array}{c}\text { Real Transcranial } \\
\text { NIR-PBMC3 }\end{array}$ & $\begin{array}{c}\text { Sham Transcranial } \\
\text { NIR-PBMC3 }\end{array}$ & $\begin{array}{c}\text { Real Transcranial } \\
\text { NIR-PBMC4 }\end{array}$ & $\begin{array}{c}\text { Sham Transcranial } \\
\text { NIR-PBMC4 }\end{array}$ \\
\hline Number of finger taps improvement (\%, Right hand) & $4.69 *$ & 1.62 & 1.53 & 2.30 \\
\hline Number of finger taps improvement (\%, Left hand) & $3.08^{*}$ & 0.12 & 3.15 & 0.04 \\
\hline
\end{tabular}

$* P<0.05$ 
thus another limitation was separation and the direct measuring of motor cortex excitability after transcranial light therapy which could be assessed by transcranial magnetic stimulation, functional magnetic resonance imaging, electroencephalogram (EEG) or other imaging instruments and it can be a complementary item for future studies. We need to mention that there was a report of superficial heating sense in many participants in real transcranial NIR-PBM groups as well as some degree of headache in two participants.

According to the results of this study, our hypothesis that transcranial light irradiation may improve the motor performance in healthy subjects is generally true. Therefore, we specifically concluded that using the transcranial NIR-PBM with a laser source on the left side of the primary motor cortex in right-handed healthy people can increase the number of finger taps in bilateral upper extremities as an indicator of motor control and motor performance improvement. Trying a similar set-up in left-handed people is recommended. We also suggest investigating the long-term effects of the transcranial NIR-PBM on human motor performance by following up the participants or repeating the transcranial NIR-PBM for several sessions. Coordination is another important parameter to assess motor performance and control. Future studies can deal with this topic by changing or modifying the motor task.

\section{Ethical Considerations}

The written informed consent was then obtained from all of the participants and the protocol was approved by the Ethics Committee of Tabriz University of Medical Sciences with a code of IR.TBZMED.REC.1396.706. In addition, this study was an experimental single-blind randomized clinical trial, registered in the Iranian Registry of Clinical Trials (IRCT) (identifier: IRCT20180110038293N1).

\section{Conflict of Interests}

The authors declare no conflict of interest.

\section{Acknowledgments}

This study was supported by Tabriz University of Medical Sciences. The authors acknowledge the participants who participated in this study.

\section{References}

1. Rojas JC, Gonzalez-Lima F. Low-level light therapy of the eye and brain. Eye Brain. 2011;3:49-67. doi:10.2147/ EB.S21391.

2. Rojas JC, Gonzalez-Lima F. Neurological and psychological applications of transcranial lasers and LEDs. Biochem Pharmacol. 2013;86(4):447-57. doi:10.1016/j. bcp.2013.06.012.

3. Salgado S, Parreira R, Ceci L, de Oliveira L, Zangaro R. Transcranial light emitting diode therapy (TCLT) and its effects on neurological disorders. J Bioeng Biomed Sci. 2015;5(1):1-5. doi:10.4172/2155-9538.1000144.
4. Wan S, Parrish JA, Anderson R, Madden M. Transmittance of nonionizing radiation in human tissues. Photochem Photobiol. 1981;34(6):679-81. doi:10.1111/j.1751-1097.1981. tb09063.x.

5. Jagdeo JR, Adams LE, Brody NI, Siegel DM. Transcranial red and near-infrared light transmission in a cadaveric model. PloS One. 2012;7(10):e47460. doi:10.1371/journal. pone.0047460.

6. Naeser MA, Hamblin MR. Potential for transcranial laser or LED therapy to treat stroke, traumatic brain injury, and neurodegenerative disease. Photomed Laser Surg. 2011;29(7):443-6. doi:10.1089/pho.2011.9908.

7. Wong-Riley MT, Bai X, Buchmann E, Whelan HT. Light-emitting diode treatment reverses the effect of TTX on cytochrome oxidase in neurons. Neuroreport. 2001;12(14):3033-7.

8. Wong-Riley MT. Cytochrome oxidase: an endogenous metabolic marker for neuronal activity. Trends Neurosci. 1989;12(3):94-101. doi:10.1016/0166-2236(89)90165-3

9. Uozumi Y, Nawashiro H, Sato S, Kawauchi S, Shima K, Kikuchi M. Targeted increase in cerebral blood flow by transcranial near-infrared laser irradiation. Lasers Surg Med. 2010;42(6):566-76. doi:10.1002/lsm.20938.

10. Hu W-P, Wang J-J, Yu C-L, Lan C-CE, Chen G-S, Yu H-S. Helium-neon laser irradiation stimulates cell proliferation through photostimulatory effects in mitochondria. J Invest Dermatol. 2007;127(8):2048-57. doi:10.1038/sj.jid.5700826.

11. Duan R, Zhu L, Liu TCY, Li Y, Liu J, Jiao J, et al. Light emitting diode irradiation protect against the amyloid beta 25-35 induced apoptosis of PC12 cell in vitro. Lasers Surg Med. 2003;33(3):199-203. doi:10.1002/lsm.10216.

12. Yang L, Tucker D, Dong Y, Wu C, Lu Y, Li Y, et al. Photobiomodulation therapy promotes neurogenesis by improving post-stroke local microenvironment and stimulating neuroprogenitor cells. Exp Neurol. 2018; 299:86-96. doi:10.1016/j.expneurol.2017.10.013.

13. Salehpour F, Farajdokht F, Cassano P, Sadigh-Eteghad $S$, Erfani M, Hamblin MR, et al. Near-infrared photobiomodulation combined with coenzyme Q10 for depression in a mouse model of restraint stress: reduction in oxidative stress, neuroinflammation, and apoptosis. Brain Res Bull. 2019;144:213-22. doi:10.1016/j. brainresbull.2018.10.010

14. Quirk BJ, Torbey M, Buchmann E, Verma S, Whelan HT. Near-infrared photobiomodulation in an animal model of traumatic brain injury: improvements at the behavioral and biochemical levels. Photomed Laser Surg. 2012;30(9):523-9. doi:10.1089/pho.2012.3261.

15. Naeser MA, Zafonte R, Krengel MH, Martin PI, Frazier J, Hamblin MR, et al. Significant improvements in cognitive performance post-transcranial, red/near-infrared lightemitting diode treatments in chronic, mild traumatic brain injury: open-protocol study. $J$ Neurotrauma. 2014;31(11):1008-17. doi:10.1089/neu.2013.3244.

16. Salgado AS, Zângaro RA, Parreira RB, Kerppers II. The effects of transcranial LED therapy (TCLT) on cerebral blood flow in the elderly women. Lasers Med Sci. 2015;30(1):339-46. doi:10.1007/s10103-014-1669-2.

17. de Sousa MVP, Ferraresi C, Kawakubo M, Kaippert B, Yoshimura EM, Hamblin MR. Transcranial low-level laser therapy $(810 \mathrm{~nm})$ temporarily inhibits peripheral 
nociception: photoneuromodulation of glutamate receptors, prostatic acid phophatase, and adenosine triphosphate. Neurophotonics. 2016;3(1):015003. doi:10.1117/1.NPh.3.1.015003.

18. Salehpour F, Mahmoudi J, Kamari F, Sadigh-Eteghad S, Rasta SH, Hamblin MR. Brain Photobiomodulation therapy: a narrative review. Mol Neurobiol. 2018;55(8):66016636. doi: 10.1007/s12035-017-0852-4.

19. Vandewalle G, Maquet P, Dijk D-J. Light as a modulator of cognitive brain function. Trends Cogn Sci. 2009;13(10):42938. doi:10.1016/j.tics.2009.07.004.

20. Barrett D, Gonzalez-Lima F. Transcranial infrared laser stimulation produces beneficial cognitive and emotional effects in humans. Neuroscience. 2013;230:13-23. doi:10.1016/j.neuroscience.2012.11.016.

21. Lewin JS, Friedman L, Wu D, Miller DA, Thompson LA, Klein SK, et al. Cortical localization of human sustained attention: detection with functional MR using a visual vigilance paradigm. J Comput Assist Tomogr. 1996;20(5):695-701.

22. Rojas JC, Bruchey AK, Gonzalez-Lima F. Low-level light therapy improves cortical metabolic capacity and memory retention. J Alzheimers Dis. 2012;32(3):741-52. doi:10.3233/ JAD-2012-120817.

23. Salehpour F, Farajdokht F, Erfani M, Sadigh-Eteghad S, Shotorbani SS, Hamblin MR, et al. Transcranial near-infrared photobiomodulation attenuates memory impairment and hippocampal oxidative stress in sleepdeprived mice. Brain Res. 2018;1682:36-43. doi: 10.1016/j. brainres.2017.12.040.

24. Wang X, Tian F, Reddy DD, Nalawade SS, Barrett DW, Gonzalez-Lima F, et al. Up-regulation of cerebral cytochrome-c-oxidase and hemodynamics by transcranial infrared laser stimulation: a broadband nearinfrared spectroscopy study. J Cereb Blood Flow Metab. 2017;37(12):3789-802. doi:10.1177/0271678X17691783.

25. Sabouri Moghadam H, Nazari M A, Jahan A, Mahmoudi J, Moghadam Salimi M. Beneficial effects of transcranial light emitting diode (LED) therapy on attentional performance: an experimental design.Iran Red Crescent Med J. 2017;19(5):e44513. doi: 10.5812/ircmj.44513.

26. Jahan A, Nazari MA, Mahmoudi J, Salehpour F, Salimi MM. Transcranial near-infrared photobiomodulation could modulate brain electrophysiological features and attentional performance in healthy young adults. Lasers Med Sci. 2019;34(6):1193-1200. doi: 10.1007/s10103-01802710-3.

27. Lee HI, Park JH, Park MY, Kim NG, Park K-J, Choi BT, et al. Pre-conditioning with transcranial low-level light therapy reduces neuroinflammation and protects bloodbrain barrier after focal cerebral ischemia in mice. Restor Neurol Neurosci. 2016;34(2):201-14. doi:10.3233/RNN150559.

28. Moro C, Torres N, El Massri N, Ratel D, Johnstone DM, Stone J, et al. Photobiomodulation preserves behaviour and midbrain dopaminergic cells from MPTP toxicity: evidence from two mouse strains. BMC Neurosci. 2013;14(1):40. doi:10.1186/1471-2202-14-40.

29. Xuan W, Vatansever F, Huang L, Wu Q, Xuan Y, Dai T, et al. Transcranial low-level laser therapy improves neurological performance in traumatic brain injury in mice: effect of treatment repetition regimen. PloS One. 2013;8(1):e53454. doi:10.1371/journal.pone.0053454.

30. Lapchak P, Salgado K, Chao C, Zivin J. Transcranial nearinfrared light therapy improves motor function following embolic strokes in rabbits: an extended therapeutic window study using continuous and pulse frequency delivery modes. Neuroscience. 2007;148(4):907-14. doi:10.1016/j. neuroscience.2007.07.002.

31. Oron A, Oron U, Streeter J, Taboada LD, Alexandrovich A, Trembovler V, et al. Low-level laser therapy applied transcranially to mice following traumatic brain injury significantly reduces long-term neurological deficits. J Neurotrauma. 2007;24(4):651-6. doi:10.1089/ neu.2006.0198.

32. Lapchak PA, Wei J, Zivin JA. Transcranial infrared laser therapy improves clinical rating scores after embolic strokes in rabbits. Stroke. 2004;35(8):1985-8. doi:10.1161/01. STR.0000131808.69640.b7

33. Chapman LJ, Chapman JP. The measurement of handedness. Brain Cogn. 1987;6(2):175-83. doi:10.1016/02782626(87)90118-7.

34. Alipour A. The reliability and validity of chapman's handedness inventory in junior high school students. Dev Psychol J. 2006;2(7):197-205.

35. Cavill S, Bryden P. Development of handedness: comparison of questionnaire and performance-based measures of preference. Brain Cogn. 2003;53(2):149-51. doi:10.1016/S0278-2626(03)00098-8.

36. Elias LJ, Saucier DM. Neuropsychology: Clinical and experimental foundations. Bacon: Pearson; 2006. Pagination

37. Saimpont A, Mercier C, Malouin F, Guillot A, Collet C, Doyon J, et al. Anodal transcranial direct current stimulation enhances the effects of motor imagery training in a finger tapping task. Eur J Neurosci. 2016;43(1):113-9. doi:10.1111/ejn.13122.

38. Schiffer F, Johnston AL, Ravichandran C, Polcari A, Teicher MH, Webb RH, et al. Psychological benefits 2 and 4 weeks after a single treatment with near infrared light to the forehead: a pilot study of 10 patients with major depression and anxiety. Behav Brain Funct. 2009;5(1):46. doi:10.1186/1744-9081-5-46.

39. Bornstein RA, Baker G, Douglass A. Short-term retest reliability of the Halstead-Reitan Battery in a normal sample. J Nerv Ment Dis. 1987;175(4):229-32. doi:10.1097/00005053-198704000-00007.

40. Strauss E, Sherman EM, Spreen O. A compendium of neuropsychological tests: Administration, norms, and commentary. Oxford: Oxford University Press; 2006.

41. De Gennaro L, Cristiani R, Bertini M, Curcio G, Ferrara $\mathrm{M}$, Fratello $\mathrm{F}$, et al. Handedness is mainly associated with an asymmetry of corticospinal excitability and not of transcallosal inhibition. Clin Neurophysiol. 2004;115(6):1305-12. doi:10.1016/j.clinph.2004.01.014.

42. Serrien DJ, Ivry RB, Swinnen SP. Dynamics of hemispheric specialization and integration in the context of motor control. Nat Rev Neurosci. 2006;7(2):160. doi:10.1038/ nrn1849.

43. Kawashima R, Inoue K, Sato K, Fukuda H. Functional asymmetry of cortical motor control in left-handed 
subjects. Neuroreport. 1997;8(7):1729-32.

44. Herron J, Galin D, Johnstone J, Ornstein RE. Cerebral specialization, writing posture, and motor control of writing in left-handers. Science. 1979;205(4412):1285-9. doi.10.1126/science.472745.

45. Sabaté M, González B, Rodríguez M. Brain lateralization of motor imagery: motor planning asymmetry as a cause of movementlateralization. Neuropsychologia.2004;42(8):10419. doi:10.1016/j.neuropsychologia.2003.12.015.

46. Kim S-G, Ashe J, Hendrich K, Ellermann JM, Merkle
$\mathrm{H}$, Ugurbil $\mathrm{K}$, et al. Functional magnetic resonance imaging of motor cortex: hemispheric asymmetry and handedness. Science. 1993;261(5121):615-7. doi:10.1126/ science. 8342027.

47. Kawashima R, Yamada K, Kinomura S, Yamaguchi T, Matsui $\mathrm{H}$, Yoshioka S, et al. Regional cerebral blood flow changes of cortical motor areas and prefrontal areas in humans related to ipsilateral and contralateral hand movement. Brain Res. 1993;623(1):33-40.doi:10.1016/0006-8993(93)90006-9. 